\title{
$d$-wave superconductivity in the frustrated two-dimensional periodic Anderson model
}

\author{
Wei $\mathrm{Wu}^{1}$ and A.-M.-S. Tremblay ${ }^{1,2, *}$ \\ ${ }^{1}$ Département de Physique and RQMP, Université de Sherbrooke, Sherbrooke, Québec J1K 2RI, Canada \\ ${ }^{2}$ Canadian Institute for Advanced Research, Toronto, Ontario M5G 1Z8, Canada \\ (Received 29 August 2014; revised manuscript received 21 November 2014; published 23 February 2015)
}

\begin{abstract}
Superconductivity in heavy-fermion materials can sometimes appear in the incoherent regime and in proximity to an antiferromagnetic quantum critical point. Here, we study these phenomena using largescale determinant quantum Monte Carlo simulations and the dynamical cluster approximation with various impurity solvers for the periodic Anderson model with frustrated hybridization. We obtain solid evidence for a $d_{x^{2}-y^{2}}$ superconducting phase arising from an incoherent normal state in the vicinity of an antiferromagnetic quantum critical point. There is a coexistence region, and the width of the superconducting dome increases with frustration. Through a study of the pairing dynamics, we find that the retarded spin fluctuations give the main contribution to the pairing glue. These results are relevant for unconventional superconductivity in the Ce-115 family of heavy fermions.
\end{abstract}

DOI: 10.1103/PhysRevX.5.011019

\section{INTRODUCTION}

$d$-wave superconductivity in proximity to a quantum critical point has been found in many compounds, such as layered and quasi-one-dimensional organic superconductors, iron pnictides, cuprate superconductors, and heavyfermion systems. In particular, many examples of quantum critical points (QCPs) in heavy-fermion materials have been found, making them an important testing ground for theories of quantum criticality in relation to superconductivity [1]. This relationship is the main problem that we consider.

Heavy-fermion behavior arises when localized $f$-electron bands are hybridized with conduction-electron bands. This hybridization leads to the Ruderman-Kittel-KasuyaYosida (RKKY) interaction between $f$-electron local moments, and also to the eventual screening of the local moments by the Kondo effect. These competing tendencies are summarized by Doniach's phase diagram $[1,2]$ where antiferromagnetic and Fermi-liquid phases both appear. The zero-temperature transition between these phases is generally believed to be a QCP. Remarkably, superconductivity appears in the vicinity of this QCP [3], even though the nature of the QCP can change depending on the Kondo deconstruction energy scale $E_{\mathrm{loc}}^{*}$ [4-8].

A few heavy-fermion superconductors [9], for example, $\mathrm{PuCoGa}_{5}$ and $\mathrm{CeCoIn}_{5}$, show the peculiarity [1] that $d_{x^{2}-y^{2}}$

\footnotetext{
*Corresponding author.

andre-marie.tremblay@usherbrooke.ca
}

Published by the American Physical Society under the terms of the Creative Commons Attribution 3.0 License. Further distribution of this work must maintain attribution to the author(s) and the published article's title, journal citation, and DOI.
Subject Areas: Condensed Matter Physics,

Strongly Correlated Materials, Superconductivity

pairing develops out of an incoherent metallic state [10,11]. $\mathrm{CeCoIn}_{5}$ belongs to the quasi-2D Ce-115 materials that have an easily accessible transition temperature $(\sim 2 \mathrm{~K})$, below which superconducting and magnetic properties can be precisely measured [12]. They are especially interesting because the itinerant-to-localized transition of $4 f$ electrons can be readily obtained by applying pressure or changing the chemical composition [13,14]. Moreover, the observation of an evolving superconducting dome in the vicinity of the magnetic QCP strongly suggests that they are candidates for antiferromagnetic (AFM) spinfluctuation mediated superconductivity [15-17]. The proximity of antiferromagnetism to $d$-wave superconductivity is also observed in many other strongly correlated systems, such as cuprate superconductors and layered organic superconductors.

In some unusual cases [18-21], heavy-fermion superconductors have been found in the absence of an obvious nearby magnetic QCP. The americium metal under high pressure [21], the "SCII" phase of $\mathrm{CeCu}_{2} \mathrm{Si}_{2}[18,22]$, and heavily $\mathrm{Yb}$-doped $\mathrm{CeCoIn}_{5}$ [20] are examples. In an attempt to understand these superconductors, alternative scenarios have been proposed, such as the valence fluctuation hypothesis $[23,24]$, or the composite pairing theory [25-27]. Yet, up to now, no consensus has been reached. There are also materials where, despite the absence of an obvious QCP, spin-fluctuation mediated pairing is considered essential, for instance, CeIrIn 5 [28-30].

On the theoretical side, the two-dimensional periodic Anderson model (PAM), the Kondo-lattice model (KLM), or the degenerate Coqblin-Schrieffer model [31] is expected to capture the essential physics of spin-fluctuation mediated superconductivity in $\mathrm{Ce}-115$ compounds. 
Previous analytical studies include large- $N$ approaches [1], mean-field theory [32], and phenomenological models of fermions coupled to fluctuating Bose modes [33]. The PAM and KLM models have also been treated with the variational method [34], exact diagonalization, and densitymatrix renormalization group calculations on small clusters [35]. In these studies, Heisenberg exchange is usually artificially added to simulate the RKKY interaction.

Here, we show, using large-scale determinant quantum Monte Carlo (DQMC) simulations [36], as well as the dynamical cluster approximation (DCA) $[37,38]$, that $d_{x^{2}-y^{2}}$ superconductivity can arise out of an incoherent metallic phase in the frustrated PAM. Heisenberg exchange is not artificially added, it arises naturally from the PAM. We demonstrate that the width of the superconducting dome surrounding the QCP can be increased by increasing frustration. Based on the magnetic susceptibility and the anomalous self-energy, we find that the driving force for pairing in this model comes primarily from retarded antiferromagnetic spin fluctuations. This reinforces the hypothesis that this mechanism applies to Ce-115.

This paper is organized as follows. In Sec. II, we introduce the frustrated periodic Anderson model. Evidence for $d_{x^{2}-y^{2}}$ pairing is first presented in Sec. III using DQMC and DCA calculations done with a quantum Monte Carlo impurity solver [continuous time quantum Monte Carlo (CTQMC)] [39]. DCA results presented in the rest of Sec. III allow us to successively discuss quasiparticle coherence, the relation between the antiferromagnetic QCP and superconductivity, and, finally, the origin of pairing. The discussion in Sec. IV also contains material-specific comments. We conclude in Sec. V. Our model is justified in more detail in Appendix A, and additional information on the DCA method can be found in Appendix B.

\section{PERIODIC ANDERSON MODEL WITH FRUSTRATED HYBRIDIZATION}

Frustration, Kondo coupling strength, and f-orbital degeneracy determine Doniach's phase diagram of heavyfermion systems [7]. These effects are embodied in the frustrated periodic Anderson model on a two-dimensional square lattice [40], with Hamiltonian

$$
\begin{aligned}
H= & \sum_{k, \sigma} \epsilon_{k} c_{k, \sigma}^{\dagger} c_{k, \sigma}+\sum_{k, \sigma} \epsilon^{f} f_{k, \sigma}^{\dagger} f_{k, \sigma} \\
& +\sum_{k, \sigma} V_{k}\left(f_{k, \sigma}^{\dagger} c_{k, \sigma}+\text { H.c. }\right)+\sum_{i} U\left(n_{f}^{\uparrow}-\frac{1}{2}\right)\left(n_{f}^{\downarrow}-\frac{1}{2}\right),
\end{aligned}
$$

where $k, \sigma, i$ are the momentum, spin (or pseudo-spin), and lattice site indices, respectively. $n_{f}^{\sigma}$ denotes the occupation number operator for $f$ orbitals. The conduction band dispersion relation is chosen as $\epsilon_{k}=-2 t\left[\cos \left(k_{x}\right)+\cos \left(k_{y}\right)\right]$. The nearest-neigbor hopping integral $t$ is taken as the energy unit throughout this paper. We neglect the dispersion of $f$ orbitals and $f$-orbital degeneracy. The $f$ energy level $\epsilon^{f}$ is set to zero and the fillings are $\left\langle n_{f}\right\rangle \sim 1,\left\langle n_{c}\right\rangle \sim 0.9$. The strength of the Kondo coupling and of the RKKY interaction is determined by the combined effects of the $f$-c hybridization $V_{k}=V+$ $2 V^{\prime}\left[\cos \left(k_{x}\right)+\cos \left(k_{y}\right)\right]$ and $U$, the screened Coulomb repulsion between $f$ electrons. In an antiferromagnetic configuration of the conduction electrons, the on-site hybridization $V$ and the hybridization with nearestneighbors $V^{\prime}$ lead to competing effective interactions with the $f$ electron. The frustration is maximal when $V$ and $V^{\prime}$ are of the same order of magnitude, as in Ce-115 [10,41]. Further discussion of the model appears in Appendix A.

\section{RESULTS}

After we present evidence for $d_{x^{2}-y^{2}}$ pairing, we discuss the question of quasiparticle coherence, then the phase diagram, and conclude with the origin of pairing.

\section{A. $d_{x^{2}-y^{2}}$ pairing}

To reveal the many-body correlation effects on superconductivity in an unbiased way, we show the result of DQMC calculations of the pairing susceptibility $P$, defined by

$$
\begin{aligned}
P_{\alpha \beta, \gamma \delta}= & \frac{1}{N} \frac{1}{G} \sum_{i, j} \sum_{r, r^{\prime}} g\left(r^{\prime}\right) g^{*}(r) \\
& \times \int_{0}^{\beta}\left\langle d_{\alpha, j+r^{\prime}, \downarrow}(\tau) d_{\beta, j, \uparrow}(\tau) d_{\gamma, i, \uparrow}^{\dagger}(0) d_{\delta, i+r, \downarrow}^{\dagger}(0)\right\rangle d \tau,
\end{aligned}
$$

where the greek indices represent either conduction $c$ or localized $f$-electron operators, $g(r)$ is the form factor in real space, and $G=\sum_{r}|g(r)|^{2}$ is the normalization factor. Let $P^{0}$ be the bubble contribution without vertex corrections. For a given pairing channel, the sign of $P-P^{0}$ basically reflects whether the pairing is favored (positive) or not (negative) [42].

Figure 1 displays our DQMC results for $d_{x^{2}-y^{2}}, s^{-}$, and extended $s$-wave pairing susceptibilities [43] as a function of temperature $T$ for $V=0.3, V^{\prime}=0.75$, and $U=4$. We learn that among those various pairing channels, $d_{x^{2}-y^{2}}$ dominates [10] since the effective pairing interaction $P-P^{0}$ increases rapidly as $T$ is lowered (inset of Fig. 1), suggesting that a divergent susceptibility would occur at the Berezinsky-Kosterlitz-Thouless (BKT) transition temperature $T_{\mathrm{BKT}}$ [44]. This contrasts with local $s$-wave pairing where the effective interaction $P-P^{0}$ decreases, becoming more negative as $T$ is lowered. This rules out $s$-wave pairing [45].

By varying $V$ and $V^{\prime}$, the itinerant character of $f$ electrons can be adjusted. This is shown in the inset of 


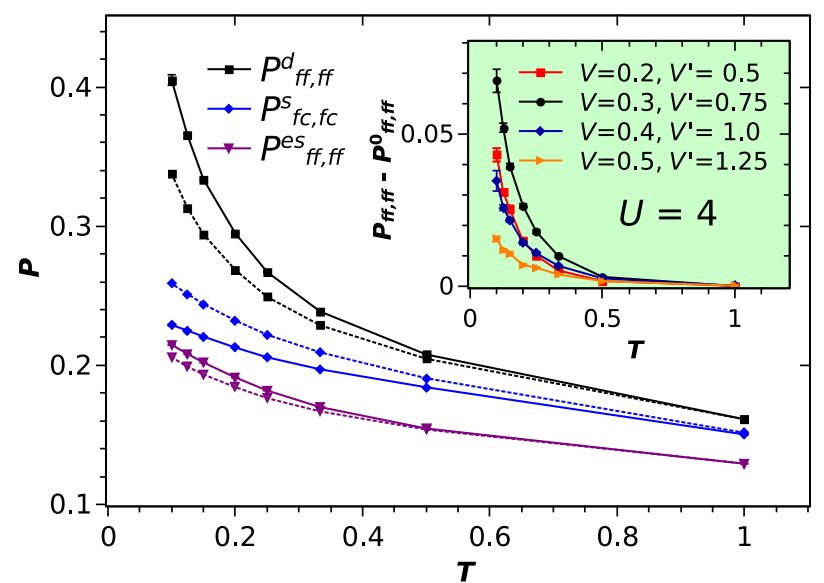

FIG. 1. Pairing susceptibilities for $V=0.3, V^{\prime}=0.75, U=4$ calculated with DQMC on a $12 \times 12 \times 2$ lattice with periodic boundary conditions. The Trotter decomposition imaginary time interval is $\Delta \tau=0.0625$. Solid curves denote susceptibilities $P$ including vertex contributions while dashed lines represent the bubble contribution $P^{0}$. The inset shows the effective pairing interaction $P-P^{0}$ for $d_{x^{2}-y^{2}}$ pairing in the $(f f, f f)$ channel, keeping the ratio $V^{\prime} / V$ constant but changing the hybridization gradually from $V=0.2, V^{\prime}=0.5$ to $V=0.5, V^{\prime}=1.25$. Increasing the hybridization makes the $f$ electrons more itinerant.

Fig. 1, where DQMC results suggest that, at fixed $V^{\prime} / V$, the pairing strength has a maximum for intermediate $V$ and $V^{\prime}$, namely, for $V=0.3$ and $V^{\prime}=0.75$. This occurs in the vicinity of an antiferromagnetic phase transition, as we see below.

Further insight into the nature of pairing and on the phase diagram is provided by DCA [37,47], which allows us to reach much lower temperature than DQMC. Following Ref. [48], the pairing susceptibilities are obtained from small pinning fields in the linear response region. The results are shown in Fig. 2 for temperatures as low as
$T \sim 0.015$. The inset of Fig. 2(a) shows that $T_{c}$ can be extrapolated from the diverging susceptibilities. Since in Ce-115 materials the Coulomb repulsion $U$ of $4 f$ orbitals is expected to be significantly larger than the local density approximation bandwidth $(U \sim 5 \mathrm{eV}$ versus $W \sim 400 \mathrm{meV}$ ) [49], we study the evolution of pairing upon approaching the extended Kondo limit of the PAM model. In Fig. 2(a), we display the $d_{x^{2}-y^{2}}$ pairing susceptibility in the $(f f, f f)$ channel, keeping $V^{2} / U$ constant at $V^{2} / U \approx 0.0225$, and increasing $U$ [50]. The behavior as a function of $T$ clearly suggests that $d_{x^{2}-y^{2}}$ pairing is stable in the extended Kondo limit. In fact, the estimated $T_{c}$ grows with increasing $U$, despite the fact that at large $T$ the susceptibility is suppressed with increasing $U$. At large $T$, where vertex corrections are not important, $U$ reduces the low-energy DOS, decreasing the pairing susceptibility. By contrast, at low $T$, increasing $U$ drastically diminishes the higher-order frustrated exchange terms, therefore enhancing the magnetic interaction vertices that mediate Cooper pairing.

Since a heavy fermion is composed of both $f$ and $c$ electrons, $a_{k \sigma}^{\dagger}=u_{k} c_{k \sigma}^{\dagger}+v_{k} f_{k \sigma}^{\dagger}$, the heavy-fermion Cooper pair $\left\langle a_{k, \uparrow}^{\dagger} a_{-k, \downarrow}^{\dagger}\right\rangle$ has four different $d_{x^{2}-y^{2}}$ susceptibilities that should diverge simultaneously at $T_{c}$. This is shown in Fig. 2(b). Since the RKKY coupling between the local moments of the $f$ orbitals is emergent in the PAM, the magnetic "pairing glue" originates from the $f$ orbitals. This is consistent with Fig. 2(b). Note that at large $T$, when Kondo screening is weak, the $(f c, f f)$ and $(c c, f f)$ channels are strongly suppressed and the effective pairings $P-P^{0}$ in the $(c c, c c)$ and $(f c, f c)$ channels, shown in the inset, are small compared with the $(f f, f f)$ channel in the inset of Fig. 1. This differs from the prediction of the composite pairing theory [25] for a two-channel Kondo lattice model. That model has a different source of pairing, leading to a dominant $f c$ pairing channel.
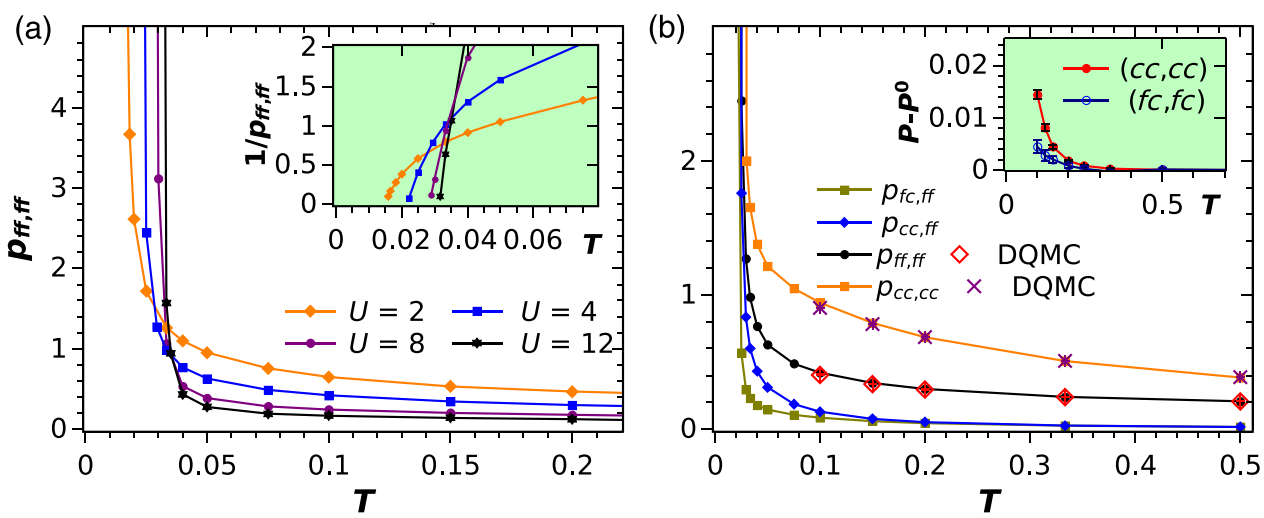

FIG. 2. $d_{x^{2}-y^{2}}$ pairing susceptibility as a function of temperature from DCA calculations on $2 \times 2 \times 2$ clusters. (a) $(f f, f f)$ channel for $U=2,4,8,12$ at constant $V^{2} / U$ and $V^{\prime}=2.5 V$. The inset shows that the inverse pairing susceptibilities extrapolate to zero, signaling that $T_{c}$ increases with $U$. (b) Comparison of different channels for $V=0.3, V^{\prime}=0.75$, and $U=4$. Available DQMC results at large $T$, shown by crosses and open diamonds, are in excellent agreement with those of DCA. The inset shows the effective pairing interaction in the $(c c, c c)$ and $(f c, f c)$ channels calculated by DQMC. They are small compared to their $(f f, f f)$ counterpart shown in the inset of Fig. 1. 


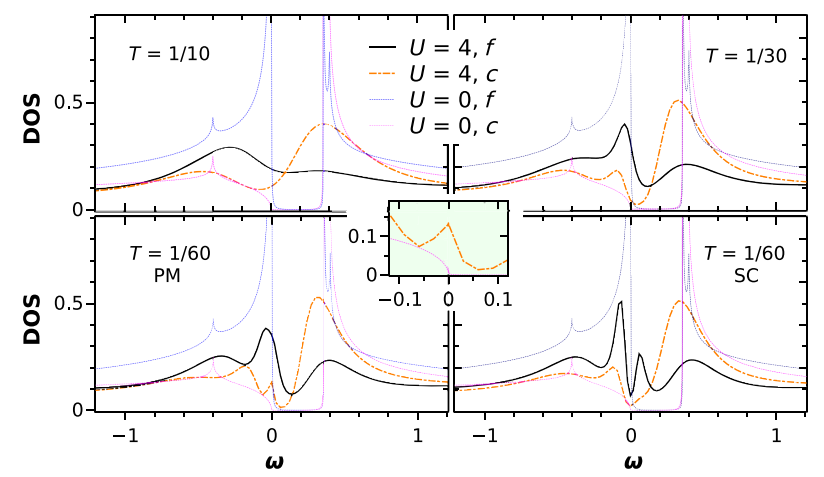

FIG. 3. Local density of states of $f$ and conduction bands at $V=0.4, V^{\prime}=0.8$, and $U=4$ for various temperatures. For reference, the DOS for the noninteracting case $(U=0)$ is repeated on all panels. The bottom left-hand panel shows the DOS when superconductivity is artificially removed. The corresponding low-energy conduction band DOS in the underlying normal state is enlarged in the inset. PM and SC in the bottom half of the figure refer respectively to paramagnetic and superconducting phases.

\section{B. Quasiparticle coherence}

We stress that complete screening of the local moments is not a necessary condition to find diverging pairing susceptibilities. In fact, we find that complete screening of $f$ moments does not occur even when the QCP is approached. This is confirmed by the fact that the magnetic susceptibility increases with decreasing $T$ (not shown), meaning that in our case the Fermi surface is small at the antiferromagnetic to paramagnetic transition.

This is further illustrated by the DOS obtained from maximum entropy analytic continuation [51] of the local Matsubara Green functions in Fig. 3. The non-interacting result, shown in light colors, is repeated for reference on all panels. As shown on the top-left panel of Fig. 3, at large $T$ the heavy-fermion quasiparticle is absent, and because of the intense scattering by $f$ local moments, the effective hybridization between the conduction band and the $f$-band is reduced, leading to a strong suppression of the hybridization gap, and to a larger DOS for the conduction band at the Fermi level (red line). This can be understood in the scenario of $f$-orbital selective Mott transition (OMST) $[52,53]$. The $d$-wave superconducting gap that appears on the emergent quasiparticle peak at low $T$ is displayed on the lower-right panel. On the bottom-left panel the superconducting order parameter is suppressed. One then sees that in the underlying normal state, the DOS of the conduction band develops a peak at the Fermi level, reflecting the increased damping of the low-energy quasiparticles on the corresponding $f$-orbital.

\section{Phase diagram, QCP and frustration}

To mimic the Doniach phase diagram, where the ratio of Kondo to RKKY couplings is the control parameter, we

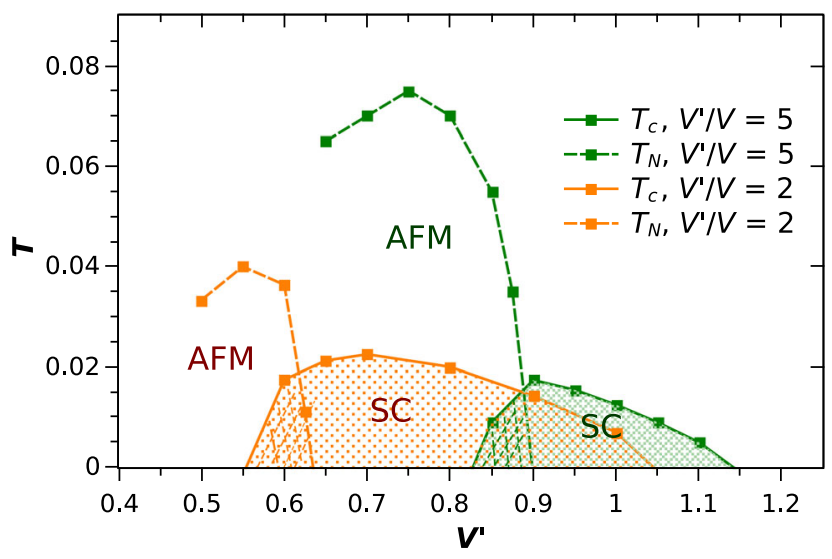

FIG. 4. Phase diagram of the frustrated 2D PAM model within $2 \times 2 \times 2$ DCA at $U=4$. Two groups of results with different $V^{\prime} / V$ ratios are shown. Darker lines (green) are drawn for the more frustrated case $\left(V^{\prime} / V=2\right)$, whereas lighter lines (orange) are for the less frustrated case $\left(V^{\prime} / V=5\right)$. The Néel temperature $T_{N}$ (dashed lines) and the superconducting $T_{c}$ (full lines) are drawn where the respective order parameters vanish. For both values of $V^{\prime} / V$ there is a uniform coexistence region indicated by crosshatch.

plot in Fig. 4 the phase boundaries in the $T-V^{\prime}$ plane for two fixed values of the frustration, $V^{\prime} / V=2$ and $V^{\prime} / V=5$. In the limit of small hybridization, the Kondo screening and antiferromagnetic RKKY correlations in the PAM/KLM both vanish, leaving only local moment fluctuations in the orbital-selective Mott insulator with large entropy at non-zero temperatures [52]. As $V^{\prime}$ increases, the Kondo energy scale and RKKY interaction both increase. First, RKKY dominates over Kondo screening and the antiferromagnetic ground state appears in coexistence with superconductivity. Increasing $V^{\prime}$ again drives the system across the QCP. Then, long-range RKKY correlations are gradually quenched by Kondo screening, and eventually superconductivity is destroyed when $f$ electrons become too itinerant.

It is striking that the superconducting dome follows the change in the position of QCP with changing magnetic frustration $\left(V^{\prime} / V\right.$ ratio). This result vividly illustrates the intrinsic connection between the QCP and superconductivity in the PAM model.

\section{Retardation and origin of pairing}

The origin of pairing may be deduced from the dynamical processes entering the real part of the anomalous self-energy $I_{\Sigma}(\omega)$ [54] at zero frequency and from the cumulative order parameter $I_{G}(\omega)$ [55], defined, respectively, by

$I_{\Sigma}(\omega)=\frac{\frac{2}{\pi} \int_{0}^{\omega} \frac{\Sigma_{a}^{\prime \prime}\left(\omega^{\prime}\right)}{\omega^{\prime}} d w^{\prime}}{\frac{2}{\pi} \int_{0}^{\infty} \frac{\Sigma_{a}^{\prime \prime}\left(\omega^{\prime}\right)}{\omega^{\prime}} d w^{\prime}}, \quad I_{G}(\omega)=\int_{0}^{\omega} \frac{d \omega^{\prime}}{\pi} F^{\prime \prime}\left(\omega^{\prime}\right)$, 

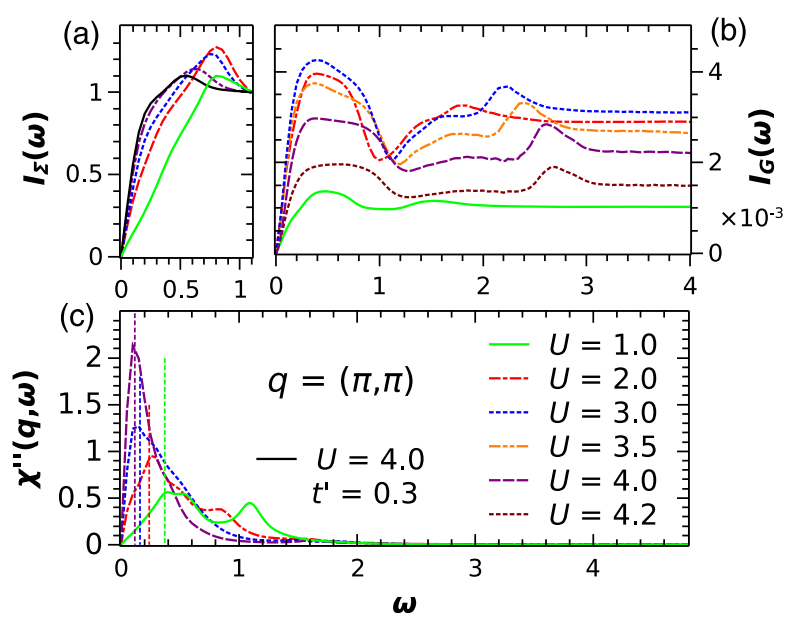

FIG. 5. (a) The low-energy part of $I_{\Sigma}(\omega)$, (b) $I_{G}(\omega)$, and (c) the imaginary part of the antiferromagnetic susceptibility $\chi_{f f, f f^{\prime \prime}}(q, \omega)$ as a function of real frequency $\omega$ for various $U$. Results are obtained with $2 \times 2 \times 2$ DCA, using a Lanczos impurity solver. The broadening factor is chosen as $\eta=0.125$. Vertical dashed lines in (c) show energies where $I_{\Sigma}(\omega)$ reaches half of its maximum value for $U=1,2,3,4$ at fixed frustration and Kondo coupling: $V=0.3$ and $V^{\prime}=0.6$.

where $\Sigma_{a}^{\prime \prime}(\omega)$ is the imaginary part of the anomalous self-energy $\sum_{a}^{i, i+r}(\omega)$ while $F^{\prime \prime}(\omega)$ is the imaginary part of the retarded lattice Gorkov function $F^{\prime \prime}(\omega)=$ $-\operatorname{Im} \int_{0}^{\beta} d \tau\left\langle c_{i+r}(\tau) c_{i}(0)\right\rangle e^{i(\omega+i \eta) \tau}$, with $i$ and $i+r$ nearest neighbors.

These functions are plotted in Fig. 5 along with the imaginary part of the antiferromagnetic spin susceptibility $\chi^{\prime \prime}(q=(\pi, \pi), \omega)$. As in the case of cuprates [54,55], we find the that the Cooper pairs initially form over an energy range comparable to that over which antiferromagnetic fluctuations develop. The dependence of pairing on the RKKY interaction strength can clearly be seen in Fig. 5, where increasing $U$, hence decreasing the RKKY interaction, shifts the peaks of both $I_{\Sigma}(\omega)$ and $\chi^{\prime \prime}[q=(\pi, \pi), \omega]$ towards the low-energy side. Frustrating magnetism in the conduction band by adding a next-nearest-neighbor hopping $t^{\prime}$ also leads to the same correlation between the two quantities: the characteristic frequency of the spin fluctuations decreases along with characteristic frequencies in both $I_{\Sigma}(\omega)$ and $I_{G}(\omega)$. Our results thus confirm that the retarded spin fluctuations mediate $d$-wave superconductivity in heavy-fermion superconductors.

In addition to the contribution of low-frequency (retarded) spin fluctuations, Fig. 5(b) shows that there can be a significant gain in pairing for an energy scale set by the upper Hubbard band of the $f$ electrons. This highfrequency (more instantaneous) contribution to pairing is much larger than what is found in the case of cuprates [55]. This is probably because the upper Hubbard band seen from the $f$-electron point of view is still in the conduction band. In other words, $I_{G}(\omega)$ is enhanced by the large
RKKY interaction [54] that results from intermediate $U$ and moderate conduction band frustration. In realistic Ce-115 materials, the RKKY interaction is believed to be small [14]; hence, the high-frequency contribution to pairing should be less important.

\section{DISCUSSION}

Spin-fluctuation mediated pairing theory finds solid support in both our DQMC and DCA results. As noted in Sec. III C, this is manifested clearly by the correlation between the location of the superconducting dome and that of the antiferromagnetic QCP when magnetic frustration $\left(V^{\prime} / V\right.$ ratio) is varied. The analysis of the frequencydependent pairing correlations based on a Lanczos exact-diagonalization solver further confirms the spinfluctuation mediated origin of superconductivity in the frustrated PAM.

Our model has a Fermi surface resembling the $\alpha$ sheet of Ce-115 materials [56], where most of the pairing occurs. In the phase diagrams, illustrated in Fig. 4, there is a coexistence region between antiferromagnetism and superconductivity, as observed in the Ce-115 family. We also note that the right-hand side of the superconducting dome does not move towards much larger $V^{\prime}$ even when the QCP does. This can be understood as indicating that, in this region, the $f$ electrons become more itinerant, leading to a suppression of antiferromagnetic fluctuations, even when there is a nearby QCP.

A few material-specific comments arise naturally. By comparing Fig. 3 with Fig. 1(c) of Ref. [57], one can verify that our model produces features in the low-energy density of states that are similar to those of $\mathrm{CeCoIn}_{5}$. The ratio of the maximum Néel temperature and the maximum superconducting $T_{c}$ for $V^{\prime} / V=2$ in Fig. 4 is similar to that observed in $\mathrm{CeRhIn}_{5}$ [58]. Finally, to explain the appearance of a superconducting phase in CeIrIn 5 , despite the absence of a nearby magnetic QCP, we note that the superconducting dome widens in parameter space when the system is more frustrated $\left(V^{\prime} / V=2\right)$, so that, in this compound, the QCP might not be observable for physically accessible parameters.

Although we expect our results to be relevant for materials where antiferromagnetic fluctuations due to localized $f$ electrons are important, there could be heavy fermion superconductors where the spin-fluctuation scenario does not apply. $\mathrm{CeCu}_{2} \mathrm{Si}_{2}$ under high ambient pressure may be an example. Indeed, recent $a b$ initio calculations [22] suggest the existence of an orbital transition in this material, which could be responsible for the underlying "SCII" phase. In other words, when two low-lying crystal-field levels become degenerate upon increasing pressure, they can compete to screen the $f$ local moment, eventually causing composite pairing $[25,26]$. To investigate cooperation and competition between composite pairing and magnetic pairing, one would need to 
consider the two-channel Kondo lattice model [59] or the two-channel periodic Anderson model.

\section{CONCLUSION}

The 2D PAM with frustration induced by the real-space structure of the hybridization $V, V^{\prime}$ between $f$ and $c$ electrons exhibits many features of the Ce- 115 materials. Our unbiased DQMC large-cluster simulations suggest that, in this model, $d_{x^{2}-y^{2}}$ pair correlations increase rapidly at low $T$ and are enhanced when the antiferromagnetic QCP is approached. The DCA-CTQMC calculations further confirm the existence of a $d_{x^{2}-y^{2}}$ superconducting phase that appears out of an incoherent metallic phase. Pairing is strongest on the $f$ electrons. In the $T-V^{\prime}$ plane, the superconducting phase has a dome shape that surrounds the QCP of the antiferromagnetic phase. Finally, through an analysis of the frequency dependence of pairing, we show that $d$-wave superconductivity in this model is mediated by retarded spin fluctuations.

\section{ACKNOWLEDGMENTS}

We thank A. Georges, M. Aichhorn, S. Burdin, P. Coleman, C. Lacroix, J. Schmalian, C. Pépin, L. Taillefer, and M. Vojta for useful discussions. We are grateful to D. Sénéchal for comments on the manuscript. This work was supported by the Natural Sciences and Engineering Research Council of Canada (NSERC), and by the Tier I Canada Research Chair Program (A.-M. S. T.). Simulations were performed on computers provided by Canadian Foundation for Innovation (CFI), Ministère de l'Éducation des Loisirs et du Sport (MELS), Calcul Québec, and Compute Canada.

\section{APPENDIX A: HYBRIDIZATION GAP AND FURTHER JUSTIFICATIONS OF THE FRUSTRATED 2D PERIODIC ANDERSON MODEL}

The dispersion relation for the PAM model of in Eq. (1) reads

$$
E_{k}^{ \pm}=\epsilon_{k}^{c} / 2 \pm \sqrt{\left(\epsilon_{k}^{c} / 2\right)^{2}+V_{k}^{2}}
$$

where $\epsilon_{k}^{c}$ is the conduction band dispersion and $V_{k}$ is the $k$-dependent hybridization between the $f$ band and the conduction band. To incorporate frustration, $V_{k}$ includes both on-site hybridization $V$ and nearest-neighbor hybridization $V^{\prime}$. When $V$ is not too large compared to $V^{\prime}$, say, $|V|<4\left|V^{\prime}\right|$, as in this paper, the hybridization gap $\Delta$,

$$
\Delta=\left|\frac{4 V V^{\prime}}{1+4 V^{\prime 2}}\right|,
$$

lies above (or below, depending on the sign of $V / V^{\prime}$ ) the Fermi level. Note that the superconducting and

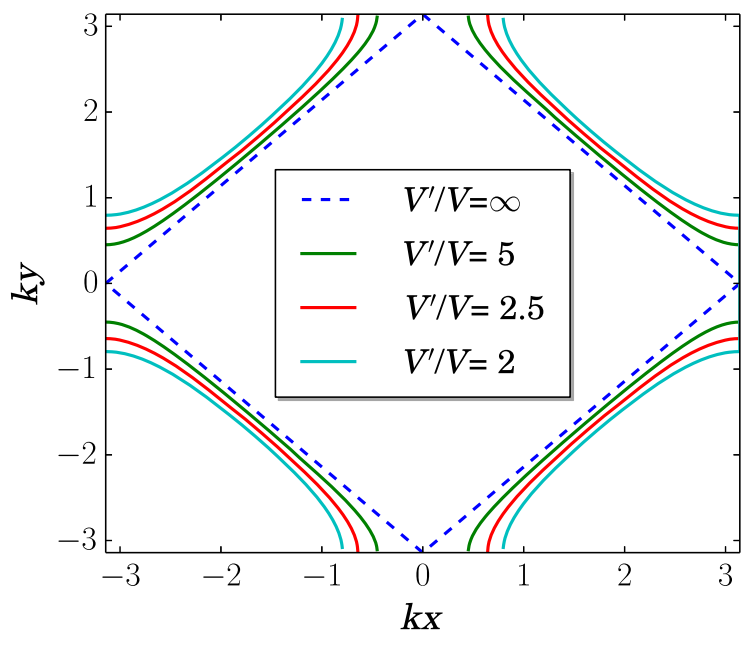

FIG. 6. Fermi surfaces of the 2D PAM model for different $V^{\prime} / V$ ratios.

hybridization gaps, as seen in the low-energy density of states, Fig. 3, are qualitatively similar to those of the experimental results displayed in Fig. 1(c) of Ref. [57].

The Fermi surfaces and band structure in the noninteracting limit are depicted, respectively, in Figs. 6 and 7. The Fermi surface resembles the $\alpha$ band of the Ce-115 materials. Pairing occurs mostly in that band in these superconductors.

The frustrated PAM is a simplified version of the effective Hamiltonian of $\mathrm{CeCoIn}_{5}$ found in Ref. [10] by fitting quasiparticle interference experiments. That Hamiltonian has 14 parameters. The main lesson we learn from it is that the $f$-c hybridization is highly frustrated since it is of the same order of magnitude for the on-site, near-neighbor, and next-nearest-neighbor terms. Similarly, the two-fluid model in Ref. [41] introduces a RKKY coupling of the form $J_{n}\left[\cos \left(k_{x}\right)+\cos \left(k_{y}\right)\right]+J_{n n} \cos \left(k_{x}\right) \cos \left(k_{y}\right)$, which carries the same general effect as our $V^{\prime}$, namely, an angular dependence related to the square-lattice symmetry.

Note that the hybridization term $V^{\prime}\left[\cos \left(k_{x}\right)+\cos \left(k_{y}\right)\right]$ is even under inversion symmetry, like the analogous term in

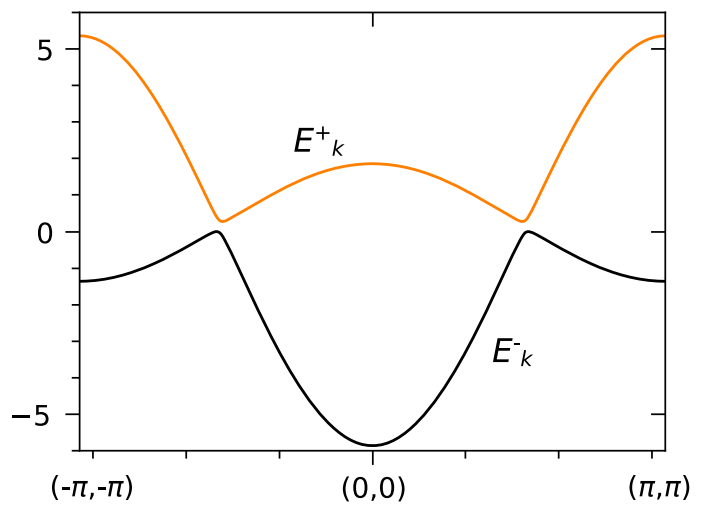

FIG. 7. Band structure of the 2D PAM for $V=0.3$ and $V^{\prime}=0.75$. 
Ref. [10]. To understand this symmetry in the Ce-115 materials, it suffices to note that the $\mathrm{Ce} f$ orbitals, which are odd under parity, couple to the out-of-plane nearestneighbor $p$ orbitals of indium, which are also odd $[13,60]$.

\section{APPENDIX B: DCA METHOD}

Throughout this paper, the DCA calculations are performed for a $2 \times 2 \times 2$ cluster, with a weak-coupling continuous time quantum Monte Carlo (CTQMC) impurity solver [39]. In a typical DCA loop, it takes about $5 \times 10^{7}$ CTQMC sweeps to calculate the Green functions. The Lanczos solver at zero temperature on a $2 \times 2$ cluster with 7-8 bath sites is used in the section where the realfrequency functions $I_{\Sigma}(\omega)$ and $I_{G}(\omega)$ are computed. Cellular Dynamical Mean-Field Theory (CDMFT) on $2 \times$ $2 \times 2$ cluster has also been carried out to compare with DCA results. Qualitative consistency is obtained, though the superconducting transition $T_{c}$ in CDMFT is lower than that obtained in DCA. This may be because DCA uses periodic boundary conditions while CDMFT does not $[37,61,62]$.

In order to calculate the pairing susceptibilities, we use the pinning field approach [48]; i.e., we observe the response of the system as small pairing fields are applied. To make sure that the response resides in the linear region, we use pinning fields of three different strengths, $5 \times 10^{-5}$, $2 \times 10^{-4}$, and $1 \times 10^{-3}$, in order to monitor the changes of the pair response function.

[1] P. Coleman, in Handbook of Magnetism and Advanced Magnetic Materials, edited by H. Kronmuller and S. Parkin (John Wiley and Sons, New York, 2007).

[2] S. Doniach, The Kondo Lattice and Weak Antiferromagnetism, Physica (Amsteram) 91B, 169 (1977); For reviews, see A. C. Hewson, The Kondo Problem to Heavy Fermions (Cambridge University Press, Cambridge, England, 1997).

[3] F. Steglich, O. Stockert, S. Wirth, C. Geibel, H. Q. Yuan, S Kirchner, and Q. Si, Routes to Heavy-Fermion Superconductivity, J. Phys. Conf. Ser. 449, 012028 (2013); For a nontrivial example of Kondo-breakdown QCP induced superconductivity, see T. Park, F. Ronning, H. Q. Yuan, M. B. Salamon, R. Movshovich, J. L. Sarrao, and J. D. Thompson, Hidden Magnetism and Quantum Criticality in the Heavy Fermion Superconductor CeRhIn ${ }_{5}$, Nature (London) 440, 65 (2006); H. Shishido, R. Settai, H. Harima, and Y. Onuki, A Drastic Change of the Fermi Surface at a Critical Pressure in $\mathrm{CeRhIn}_{5}$ : dHvaA Study under Pressure, J. Phys. Soc. Jpn. 74, 1103 (2005).

[4] F. M. Grosche, P. Agarwal, S. R. Julian, N. J. Wilson, R. K. W. Haselwimmer, S. J. S. Lister, N. D. Mathur, F. V. Carter, S.S. Saxena, and G. G. Lonzarich, Anomalous Low Temperature States in $\mathrm{CeNi}_{2} \mathrm{Ge}_{2}$ and $\mathrm{CePd}_{2} \mathrm{Si}_{2}$, J. Phys. Condens. Matter 12, L533 (2000).

[5] T. Moriya and A. Kawabata, Effect of Spin Fluctuations on Itinerant Electron Ferromagnetism, J. Phys. Soc. Jpn. 34, 639 (1973); For a review of the Hertz-Millis-Moriya theory, see H. v. Löhneysen, A. Rosch, M. Vojta, and P. Wölfle, Fermi-Liquid Instabilities at Magnetic Quantum Phase Transitions, Rev. Mod. Phys. 79, 1015 (2007).

[6] A. Schröder, G. Aeppli, E. Bucher, R. Ramazashvili, and P. Coleman, Scaling of Magnetic Fluctuations near a Quantum Phase Transition, Phys. Rev. Lett. 80, 5623 (1998); A. Schröder, G. Aeppli, R. Coldea, M. Adams, O. Stockert, H. v. Löhneysen, E. Bucher, R. Ramazashvili, and P. Coleman, Onset of Antiferromagnetism in HeavyFermion Metals, Nature (London) 407, 351 (2000).

[7] P. Coleman, Theories of Non-Fermi Liquid Behavior in Heavy Fermions, Physica (Amsterdam) 259-261B, 353 (1999); Q. Si, S. Rabello, K. Ingersent, and J. L. Smith, Locally Critical Quantum Phase Transitions in Strongly Correlated Metals, Nature (London) 413, 804 (2001); I. Paul, C. Pépin, and M. R. Norman, Kondo Breakdown and Hybridization Fluctuations in the Kondo-Heisenberg Lattice, Phys. Rev. Lett. 98, 026402 (2007); P. Gegenwart, Q. Si, and F. Steglich, Quantum Criticality in HeavyFermion Metals, Nat. Phys. 4, 186 (2008); Q. Si and F. Steglich, Heavy Fermions and Quantum Phase Transitions, Science 329, 1161 (2010); Q. Si, J. H. Pixley, E. Nica, S. J. Yamamoto, P. Goswami, R. Yu, and S. Kirchner, Kondo Destruction and Quantum Criticality in Kondo Lattice Systems, J. Phys. Soc. Jpn. 83, 061005 (2014).

[8] P. Sun and G. Kotliar, Understanding the Heavy Fermion Phenomenology from a Microscopic Model, Phys. Rev. Lett. 95, 016402 (2005).

[9] C. Pfleiderer, Superconducting Phases of f-Electron Compounds, Rev. Mod. Phys. 81, 1551 (2009).

[10] K. Izawa, H. Yamaguchi, Y. Matsuda, H. Shishido, R. Settai, and Y. Onuki, Angular Position of Nodes in the Superconducting Gap of Quasi-2D Heavy-Fermion Superconductor CeCoIn ${ }_{5}$, Phys. Rev. Lett. 87, 057002 (2001); M. P. Allan, F. Massee, D. K. Morr, J. Van Dyke, A. W. Rost, A. P. Mackenzie, C. Petrovic, and J. C. Davis, Imaging Cooper Pairing of Heavy Fermions in $\mathrm{CeCoIn}_{5}$, Nat. Phys. 9, 468 (2013); B. B. Zhou, S. Misra, E. H. da Silva Neto, P. Aynajian, R. E. Baumbach, J. D. Thompson, E. D. Bauer, and A. Yazdani, Visualizing Nodal Heavy Fermion Superconductivity in $\mathrm{CeCoIn}_{5}$, Nat. Phys. 9, 474 (2013); V. A. Sidorov, M. Nicklas, P. G. Pagliuso, J. L. Sarrao, Y. Bang, A. V. Balatsky, and J. D. Thompson, Superconductivity and Quantum Criticality in $\mathrm{CeCoIn}_{5}$, Phys. Rev. Lett. 89, 157004 (2002).

[11] D. Daghero, M. Tortello, G. A. Ummarino, J.-C. Griveau, E. Colineau, R. Eloirdi, A. B. Shick, J. Kolorenc, A. I. Lichtenstein, and R. Caciuffo, Strong-Coupling $d$-Wave Superconductivity in $\mathrm{PuCoGa}_{5}$ Probed by Point-Contact Spectroscopy, Nat. Commun. 3, 786 (2012).

[12] H. Hegger, C. Petrovic, E. G. Moshopoulou, M. F. Hundley, J. L. Sarrao, Z. Fisk, and J. D. Thompson, Pressure-Induced Superconductivity in Quasi-2D CeRhIn ${ }_{5}$, Phys. Rev. Lett. 84, 4986 (2000); C. Petrovic, P. G. Pagliuso, M. F. Hundley, R. Movshovich, J. L. Sarrao, J. D. Thompson, Z. Fisk, and P. Monthoux, Heavy-Fermion Superconductivity in $\mathrm{CeCoIn}_{5}$ at 2.3 k, J. Phys. Condens. Matter 13, L337 (2001); C. Petrovic, R. Movshovich, M. Jaime, P. G. Pagliuso, M. F. Hundley, J. L. Sarrao, Z. Fisk, and J. D. Thompson, A New Heavy-Fermion Superconductor 
CeIrIn $_{5}$ : A Relative of the Cuprates?, Europhys. Lett. 53, 354 (2001); N. J. Curro, T. Caldwell, E. D. Bauer, L. A. Morales, M. J. Graf, Y. Bang, A. V. Balatsky, J. D. Thompson, and J. L. Sarrao, Unconventional Superconductivity in $\mathrm{PuCoGa}_{5}$, Nature (London) 434, 622 (2005).

[13] K. Haule, C.-H. Yee, and K. Kim, Dynamical Mean-Field Theory within the Full-Potential Methods: Electronic Structure of $\mathrm{CeIrIn}_{5}, \mathrm{CeCoIn}_{5}$, and $\mathrm{CeRhIn}_{5}$, Phys. Rev. B 81, 195107 (2010).

[14] J. L. Sarrao and J. D. Thompson, Superconductivity in Cerium- and Plutonium-Based '115' Materials, J. Phys. Soc. Jpn. 76, 051013 (2007).

[15] M. T. Beal-Monod, C. Bourbonnais, and V. J. Emery, Possible Superconductivity in Nearly Antiferromagnetic Itinerant Fermion Systems, Phys. Rev. B 34, 7716 (1986); D. J. Scalapino, E. Loh, and J. E. Hirsch, d-Wave Pairing near a Spin-Density-Wave Instability, Phys. Rev. B 34, 8190 (1986); K. Miyake, S. Schmitt-Rink, and C. M. Varma, Spin-Fluctuation-Mediated Even-Parity Pairing in Heavy-Fermion Superconductors, Phys. Rev. B 34, 6554 (1986).

[16] T. Hu, H. Xiao, T. A. Sayles, M. Dzero, M. B. Maple, and C. C. Almasan, Strong Magnetic Fluctuations in a Superconducting State of $\mathrm{CeCoIn}_{5}$, Phys. Rev. Lett. 108, 056401 (2012).

[17] J. Van Dyke, F. Massee, M. P. Allan, J. C. Davis, C. Petrovic, and D. K. Morr, Direct Evidence for a Magnetic f-Electron Mediated Cooper Pairing Mechanism of Heavy Fermion Superconductivity in $\mathrm{CeCoIn}_{5}$, arXiv:1405.5883.

[18] H. Q. Yuan, F. M. Grosche, M. Deppe, C. Geibel, G. Sparn, and F. Steglich, Observation of Two Distinct Superconducting Phases in $\mathrm{CeCu}_{2} \mathrm{Si}_{2}$, Science 302, 2104 (2003).

[19] M. Nicklas, V. A. Sidorov, H. A. Borges, P. G. Pagliuso, J. L. Sarrao, and J. D. Thompson, Two Superconducting Phases in $\mathrm{CeRh}_{1-x} \mathrm{Ir}_{x} \mathrm{In}_{5}$, Phys. Rev. B 70, 020505 (2004).

[20] L. Shu, R. E. Baumbach, M. Janoschek, E. Gonzales, K. Huang, T. A. Sayles, J. Paglione, J. O'Brien, J. J. Hamlin, D. A. Zocco, P.-C. Ho, C. A. McElroy, and M. B. Maple, Correlated Electron State in $\mathrm{Ce}_{1-x} \mathrm{Yb}_{x} \mathrm{CoIn}_{5}$ Stabilized by Cooperative Valence Fluctuations, Phys. Rev. Lett. 106, 156403 (2011).

[21] J.-C. Griveau, J. Rebizant, G. H. Lander, and G. Kotliar, Superconductivity in the Americium Metal as a Function of Pressure: Probing the Mott Transition, Phys. Rev. Lett. 94, 097002 (2005); S. Savrasov, K. Haule, and G. Kotliar, Many-Body Electronic Structure of Americium Metal, Phys. Rev. Lett. 96, 036404 (2006).

[22] L. V. Pourovskii, P. Hansmann, M. Ferrero, and A. Georges, Theoretical Prediction and Spectroscopic Fingerprints of an Orbital Transition in $\mathrm{CeCu}_{2} \mathrm{Si}_{2}$, Phys. Rev. Lett. 112, 106407 (2014).

[23] K. Miyake, O. Narikiyo, and Y. Onishi, Superconductivity of Ce-Based Heavy Fermions under Pressure: Valence Fluctuation Mediated Pairing Associated with Valence Instability of Ce, Physica (Amsterdam) 259B, 676 (1999).

[24] E. D. Bauer, M. M. Altarawneh, P. H. Tobash, K. Gofryk, O. E. Ayala-Valenzuela, J. N. Mitchell, R. D. McDonald, C. H. Mielke, F. Ronning, J.-C. Griveau, E. Colineau, R. Eloirdi, R. Caciuffo, B. L. Scott, O. Janka, S. M. Kauzlarich, and J. D. Thompson, Localized $5 f$ Electrons in Superconducting $\mathrm{PuCoIn}_{5}$ : Consequences for Superconductivity in $\mathrm{PuCoGa}_{5}$, J. Phys. Condens. Matter 24, 052206 (2012).

[25] R. Flint, M. Dzero, and P. Coleman, Heavy Electrons and the Symplectic Symmetry of Spin, Nat. Phys. 4, 643 (2008).

[26] R. Flint and P. Coleman, Tandem Pairing in Heavy-Fermion Superconductors, Phys. Rev. Lett. 105, 246404 (2010).

[27] O. Erten, R. Flint, and P. Coleman, Molecular Pairing and Fully Gapped Superconductivity in Yb-doped $\mathrm{CeCoIn}_{5}$, Phys. Rev. Lett. 114, 027002 (2015).

[28] Y. Kasahara, T. Iwasawa, Y. Shimizu, H. Shishido, T. Shibauchi, I. Vekhter, and Y. Matsuda, Thermal Conductivity Evidence for a $d_{x^{2}-y^{2}}$ Pairing Symmetry in the HeavyFermion CeIrIn ${ }_{5}$ Superconductor, Phys. Rev. Lett. 100, 207003 (2008).

[29] T. Shang, R. E. Baumbach, K. Gofryk, F. Ronning, Z. F. Weng, J. L. Zhang, X. Lu, E. D. Bauer, J. D. Thompson, and H. Q. Yuan, CeIrIn ${ }_{5}$ : Superconductivity on a Magnetic Instability, Phys. Rev. B 89, 041101 (2014).

[30] Y.-f. Yang, D. Pines, and N. J. Curro, Scaling and Superconductivity in Heavy Electron Materials, arXiv:1410.0452.

[31] T. M. Rice and K. Ueda, Gutzwiller Variational Approximation to the Heavy-Fermion Ground State of the Periodic Anderson Model, Phys. Rev. Lett. 55, 995 (1985).

[32] Y. Liu, H. Li, G.-M. Zhang, and L. Yu, d-Wave Superconductivity Induced by Short-Range Antiferromagnetic Correlations in the Two-Dimensional Kondo Lattice Model, Phys. Rev. B 86, 024526 (2012).

[33] S. Nishiyama, K. Miyake, and C. M. Varma, Superconducting Transition Temperatures for Spin-Fluctuation Superconductivity: Application to Heavy-Fermion Compounds, Phys. Rev. B 88, 014510 (2013).

[34] M. Z. Asadzadeh, M. Fabrizio, and F. Becca, Superconductivity from Spoiling Magnetism in the Kondo Lattice Model, Phys. Rev. B 90, 205113 (2014).

[35] J. C. Xavier and E. Dagotto, Robust d-Wave Pairing Correlations in the Heisenberg Kondo Lattice Model, Phys. Rev. Lett. 100, 146403 (2008).

[36] R. Blankenbecler, D. J. Scalapino, and R. L. Sugar, Monte Carlo Calculations of Coupled Boson-Fermion Systems. I, Phys. Rev. D 24, 2278 (1981).

[37] T. Maier, M. Jarrell, T. Pruschke, and M. H. Hettler, Quantum Cluster Theories, Rev. Mod. Phys. 77, 1027 (2005).

[38] Further details on the methodology can be found in Appendix B.

[39] A. N. Rubtsov, V. V. Savkin, and A. I. Lichtenstein, Continuous-Time Quantum Monte Carlo Method for Fermions, Phys. Rev. B 72, 035122 (2005).

[40] H. Weber and M. Vojta, Heavy-Fermion Metals with Hybridization Nodes: Unconventional Fermi Liquids and Competing Phases, Phys. Rev. B 77, 125118 (2008).

[41] Y.-f. Yang and D. Pines, Emergent States in Heavy-Electron Materials, Proc. Natl. Acad. Sci. U.S.A. 109, 18247 (2012).

[42] D. J. Scalapino, in Handbook of High-Temperature Superconductivity, edited by J. Robert Schrieffer (Springer, New York 2007), Chap. 13.

[43] The form factors for $d_{x^{2}-y^{2}}, s$, and extended $s$ wave in $k$ space are, respectively, $\cos (k x)-\cos (k y), 1$ and $\cos (k x)+\cos (k y)$. 
[44] T. A. Maier, M. Jarrell, T. C. Schulthess, P. R. C. Kent, and J. B. White, Systematic Study of d-Wave Superconductivity in the 2D Repulsive Hubbard Model, Phys. Rev. Lett. 95, 237001 (2005).

[45] Such pairing is seen for the KLM [46] that does not have the nearest-neigbor hybridization.

[46] O. Bodensiek, R. Žitko, M. Vojta, M. Jarrell, and T. Pruschke, Unconventional Superconductivity from Local Spin Fluctuations in the Kondo Lattice, Phys. Rev. Lett. 110, 146406 (2013).

[47] M. H. Hettler, A. N. Tahvildar-Zadeh, M. Jarrell, T. Pruschke, and H. R. Krishnamurthy, Nonlocal Dynamical Correlations of Strongly Interacting Electron Systems, Phys. Rev. B 58, R7475 (1998).

[48] N. Lin, E. Gull, and A. J. Millis, Two-Particle Response in Cluster Dynamical Mean-Field Theory: Formalism and Application to the Raman Response of HighTemperature Superconductors, Phys. Rev. Lett. 109, 106401 (2012).

[49] J. Costa-Quintana and F. López-Aguilar, Electronic Structure of $\mathrm{CeCoIn}_{5}$, Phys. Rev. B 67, 132507 (2003).

[50] R. Dong, J. Otsuki, and S. Y. Savrasov, Scaling between Periodic Anderson and Kondo Lattice Models, Phys. Rev. B 87, 155106 (2013).

[51] M. Jarrell and J. E. Gubernatis, Bayesian Inference and the Analytic Continuation of Imaginary-Time Quantum Monte Carlo Data, Phys. Rep. 269, 133 (1996).

[52] L. De Leo, M. Civelli, and G. Kotliar, T=0 HeavyFermion Quantum Critical Point as an Orbital-Selective Mott Transition, Phys. Rev. Lett. 101, 256404 (2008).

[53] M. Vojta, Orbital-Selective Mott Transitions: Heavy Fermions and Beyond, J. Low Temp. Phys. 161, 203 (2010).
[54] T. A. Maier, D. Poilblanc, and D. J. Scalapino, Dynamics of the Pairing Interaction in the Hubbard and $t$-J Models of High-Temperature Superconductors, Phys. Rev. Lett. 100, 237001 (2008).

[55] B. Kyung, D. Sénéchal, and A.-M. S. Tremblay, Pairing Dynamics in Strongly Correlated Superconductivity, Phys. Rev. B 80, 205109 (2009).

[56] P. Aynajian, E. H. da Silva Neto, A. Gyenis, R. E. Baumbach, J. D. Thompson, Z. Fisk, E. D. Bauer, and A. Yazdani, Visualizing Heavy Fermions Emerging in a Quantum Critical Kondo Lattice, Nature (London) 486, 201 (2012).

[57] B. B. Zhou, S. Misra, E. H. da Silva Neto, P. Aynajian, R. E. Baumbach, J. D. Thompson, E. D. Bauer, and A. Yazdani, Visualizing Nodal Heavy Fermion Superconductivity in $\mathrm{CeCoIn}_{5}$, Nat. Phys. 9, 474 (2013).

[58] M. Yashima, S. Kawasaki, H. Mukuda, Y. Kitaoka, H. Shishido, R. Settai, and Y.Ōnuki, Quantum Phase Diagram of Antiferromagnetism and Superconductivity with a Tetracritical Point in CeRhin ${ }_{5}$ in Zero Magnetic Field, Phys. Rev. B 76, 020509 (2007).

[59] M. Jarrell, H. Pang, and D. L. Cox, Phase Diagram of the TwoChannel Kondo Lattice, Phys. Rev. Lett. 78, 1996 (1997).

[60] J. H. Shim, K. Haule, and G. Kotliar, Modeling the Localized-to-Itinerant Electronic Transition in the Heavy Fermion System CeIrIn5, Science 318, 1615 (2007).

[61] G. Kotliar, S. Y. Savrasov, K. Haule, V. S. Oudovenko, O. Parcollet, and C.A. Marianetti, Electronic Structure Calculations with Dynamical Mean-Field Theory, Rev. Mod. Phys. 78, 865 (2006).

[62] A. M. S. Tremblay, B. Kyung, and D. Sénéchal, Pseudogap and High-Temperature Superconductivity from Weak to Strong Coupling. Towards a Quantitative Theory, Low Temp. Phys. 32, 424 (2006). 